\title{
ON THE COMPACTIFICATION OF ARITHMETICALLY DEFINED QUOTIENTS OF BOUNDED SYMMETRIC DOMAINS
}

\author{
BY W. L. BAILY, JR. AND A. BOREL ${ }^{1}$
}

Communicated by Eldon Dyer, March 23, 1964

In previous papers [13], [2], [16], [3], [11], the theory of automorphic functions for some classical discontinuous groups $\Gamma$, such as the Siegel or Hilbert-Siegel modular groups, acting on certain bounded symmetric domains $X$, has been developed through the construction of a natural compactification of $X / \Gamma$, which is a normal analytic space, projectively embeddable by means of automorphic forms. The purpose of this note is to announce similar results for general arithmetic groups, which include the earlier ones as special cases.

1. For algebraic groups, we follow the notation and conventions of [4], [5]. $G \subset G L(m, C)$ will be a semisimple linear algebraic group defined over $Q$, and, for every subring $B$ of $C$, we put as usual $G_{B}=$ $G \cap G L(m, B)$. We let $\Gamma$ be an arithmetic subgroup of $G$, i.e., a subgroup of $G_{Q}$ commensurable with the group $G_{Z}$ of units of $G$. The group acts on the right, in a properly discontinuous manner, on the symmetric space $X=K \backslash G_{R}$, where $K$ is a maximal compact subgroup of $G_{R}$. Here we assume $X$ to be hermitian symmetric, and therefore [7] equivalent to a bounded symmetric domain. The quotient $V=X / \Gamma$ then carries a natural ringed structure with which it becomes an irreducible normal analytic space.

There is no loss in generality in assuming $G$ to be connected and centerless, and we shall do so. Although this is not essential, we shall here for simplicity assume that $G$ is simple over $\boldsymbol{Q}$, in other words, that it has no proper invariant subgroup $N \neq(e)$ defined over $\boldsymbol{Q}$. We are, of course, interested here only in the case where $V$ is not compact; this means that $G_{R}$ has no compact factor $\neq(e)$ and that $G$ contains a torus $S \neq(e)$ which splits over $\boldsymbol{Q}$ [5].

2. Rational boundary components. The space $X$ has a "natural" compactification, obtained by taking the closure $\bar{X}$ of $X$ in the Harish-Chandra realization of $X$ as a bounded symmetric domain [7], [8]. The boundary is then the union of finitely many orbits of $G_{R}$, each of which has a fibration, whose fibers are locally closed

${ }^{1}$ Partial support by N.S.F. grant GP91 for the first-named author, by N.S.F. grant GP2403 for the second-named author. 
analytic subvarieties of the ambient complex vector space, and are symmetric bounded domains for certain subgroups of $G_{R}$ [9], [10], [11]. These fibers are the boundary components of $X$.

Definition 1. A boundary component $F$ of $X$ is called rational if: (i) The complexification $\Re(F)_{C}$ of the normalizer $\Re(F)$ $=\left\{g \in G_{R} \mid F \cdot g=F\right\}$ of $F$ is an algebraic subgroup defined over $Q$ of $G$; (ii) $\mathscr{T}(F)_{C}$ contains a normal subgroup $M^{\prime}$ defined over $Q$ such that $M_{R}^{\prime} \subset \mathbb{Z}(F)=\{g \in \mathscr{N}(F) \mid x \cdot g=x(x \in F)\}$, and that $Z(F) / M_{R}^{\prime}$ is compact.

These conditions imply readily that the image $\Gamma(F)$ of $\mathfrak{x}(F) \cap \Gamma$ in $\mathscr{T}(F) / M_{R}^{\prime}$ is an arithmetic group, acting properly discontinually on $F$. The previous definition can of course be given for any semisimple group $L$ over $Q$, and a boundary component of the corresponding symmetric space $Y$ with respect to any Satake compactification of $Y$ [14]. It is then slightly weaker than the definition used in $[4, \S 4]$. Coming back to $X$, it is known that its natural compactification (with its boundary components) is equivalent to the Satake compactification associated to a certain class of representations [10]. The proofs of the results below make strong use of that fact.

TheOREM 1. The map $F \rightarrow \mathfrak{T}(F)_{C}$ yields a 1-1 correspondence between rational boundary components and proper maximal parabolic subgroups defined over $Q$ of $G$.

We recall that an algebraic subgroup $H$ of $G$ is parabolic if $G / H$ is a projective variety. The theorem implies in particular that, in the natural compactification, condition (ii) of Definition 1 is a consequence of (i). This theorem is proved by a rather detailed investigation of the $R$-roots and $Q$-roots of $G$, and on how the former restrict on the latter, which, at some points, makes use of the classification. In particular, the following, which generalizes results of $[15, \S 5]$, is proved:

Proposition 1. Let ${ }_{Q} T$ be a maximal Q-trivial torus of $G$. Then the system ${ }_{Q} \Sigma$ of $Q$-roots (cf. [4]) is either of type $C_{s}: \pm(1 / 2)\left(\gamma_{i} \pm \gamma_{j}\right)$ $\left(1 \leqq i, j \leqq s=\operatorname{dim}_{Q} T\right)$ or of type $B C_{s}=C_{s} \cup\left\{(1 / 2) \gamma_{i}\right\}_{i_{s}}$. If we choose compatible orderings on ${ }_{R} \Sigma$ and ${ }_{Q} \Sigma$, then each simple $Q$-root is the restriction of exactly $q$ simple $R$-roots, where $q$ is the number of simple factors of $G_{R}$.

3. A topological compactification of $X / \Gamma$. Let $\Omega$ be a sufficiently large fundamental set for $\Gamma$ in $X$, as in [4, p. 31]. Using Theorem 1, it may be shown, as in [4], that the closure $\bar{\Omega}$ of $\Omega$ is contained in the union of $\Omega$ and of finitely many rational components, and meets every 
equivalence class under $\Gamma$ of rational boundary components. In what follows, we let $X^{*}$ denote the union of $X$ and of all rational boundary components. It is acted upon by $G_{Q}$. Moreover, we may apply some results of [15] and obtain the

THEOREM 2. There exists a topology on $X^{*}$, which induces the natural topology on $\Omega$, in which every element of $G_{Q}$ operates continuously, and such that $V^{*}=X^{*} / \Gamma$, endowed with the quotient topology, is a compact Hausdorff space.

The construction shows that $V^{*}=V \cup V_{1} \cup \cdots \cup V_{t}$, where $V=X / \Gamma$ is open, everywhere dense, $V_{i}=F_{i} / \Gamma\left(F_{i}\right)$, and the $F_{i}$ 's are such that the groups $\mathfrak{T}\left(F_{i}\right)_{C}$ form a system of representatives for the equivalence classes, modulo inner automorphisms by elements of $\Gamma$, of proper maximal parabolic subgroups over $\boldsymbol{Q}$ of $G$. Moreover, $V_{i}$ carries a natural structure of irreducible normal analytic space, and $\bar{V}_{\boldsymbol{i}}$ is the union of $V_{i}$ and of some $V_{j}$ 's of strictly smaller dimension $(1 \leqq i \leqq t)$. We may also prove the

Lemma. Each point $v \in V^{*}$ has a fundamental system of neighborhoods $\left\{U_{\imath}\right\}$ such that $U_{\imath} \cap V$ is connected for each $\iota$.

4. The ringed structure on $V^{*}$. A complex-valued function $f$ defined on an open subset $U$ of $V^{*}$ will be called an Je-function on $U$ if it is continuous and if its restrictions to $V \cap U$ and to $V_{i} \cap U$ are analytic in the given analytic structures $(1 \leqq i \leqq t)$. The sheaf of germs of $\mathcal{H}$-functions, to be denoted by $\mathfrak{K C}$, provides $V^{*}$ with a ringed structure. Our next goal is to prove that $\left(V^{*}, \mathfrak{H C}\right)$ is a normal analytic space, whose analytic structure is compatible with the given ones on $V$ and the $V_{i}$ 's. It is easily deduced from the above lemma (as in $[16$, Exposé 11]) that the stalk $\mathfrak{K C}_{v}$ of $\mathfrak{F}$ at $v \in V^{*}$ is integrally closed for every $v$. Using this fact, the properties of the $V_{i}$ 's stated above, the Remmert-Stein theorem on removable singularities of analytic sets [12], and arguments similar to those of [3, pp. 870-872] (see also [16, Exposé 11]), one shows that it is enough to check the two following properties of $\mathfrak{F}$ : (a) For each $i(1 \leqq i \leqq t)$, the sheaf of germs of restrictions to $V_{i}$ of $\mathfrak{H}$-functions is the structural sheaf of $V_{i}$; (b) each point $v \in V^{*}$ has a neighborhood $U_{v}$ such that the $H$-functions on $U_{v}$ separate the points of $U_{v}$. In order to do this, we study certain automorphic forms introduced below.

5. Poincarê-Eisenstein series. To construct these, we need some results of Pyateckiǐ-Shapiro [11] and of Korányi and Wolf [9] about unbounded realizations of $X$. Given a boundary component $F$ of $X$, 
there exists a "partial Cayley transform" $\nu_{F}$ of $X$ onto a Siegel domain of the third kind (in the sense of [11]), given by

$$
\operatorname{Im} z-\operatorname{Re} L_{t}(u, u) \in V \quad(t \in F)
$$

(for notation and definitions, see [11, pp. 26-35]) in such a way that the elements of $\mathfrak{l}(F)$ become quasi-linear transformations in the sense of [11], and those of $\mathcal{Z}(F)$ take the form

$$
z \rightarrow A \cdot z+a(u, t), \quad u \rightarrow B \cdot u+b(t), \quad t \rightarrow t .
$$

Assume now that $F$ is a rational boundary component. Let $J_{F}(w, g)$ be the functional determinant of $g$ at $w$ in the coordinates $(z, u, t)$ of the unbounded realization associated to $F$. It can be shown that $J_{F}\left(w, g \cdot g^{\prime}\right)=J_{F}(w, g) \cdot \chi\left(g^{\prime}\right)$ if $g^{\prime} \in \mathcal{Z}(F), \chi$ being a rational character on $Z(F)$ which takes the values \pm 1 on $M^{\prime} \cap \Gamma$. Then, given a polynomial $P$ in the coordinates $t$ on $F$, and a positive integer $m$, we may form the following series, to be called a Poincaré-Eisenstein series

$$
E_{P}(w)=\sum_{\gamma \in \Gamma /\left(\Gamma \cap M^{\prime}\right)} P(t(w \cdot \gamma)) J_{F}(w, \gamma)^{2 m},
$$

where $w=(z, u, t), t(w)=t$, and $M^{\prime}$ is the subgroup of $\mathrm{Z}(F)_{C}$ occurring in (ii) of Definition 1. It can be shown that, for sufficiently large $m$, such a series converges uniformly on every compact set. By the use of some properties of $J_{F}$, and of standard facts on Poincare series, this convergence proof is reduced to that of ordinary Eisenstein series (associated to parabolic subgroups over $\boldsymbol{Q}$ of $G$ ), where we apply an unpublished criterion of Godement. We then study the behaviour of such series as we approach a boundary component (the effect of the operator $\Phi$ of [16]). It can be shown that the "limit" of $E_{P}$ on any rational boundary component $F^{\prime}$ may be viewed as an automorphic form for $\Gamma\left(F^{\prime}\right)$, which is a Poincaré series if $F^{\prime}$ is equivalent to $F$, and is zero if $\operatorname{dim} F^{\prime} \leqq \operatorname{dim} F$, and $F^{\prime}$ is not equivalent under $\Gamma$ to $F$. In particular, every Poincaré series with respect to the bounded realization of $X$ is a cusp form (i.e., has limit zero on all proper rational boundary components).

6. The main results. These facts, and well-known properties of Poincare series [6], [16], show that the sheaf $\operatorname{TC}$ verifies the conditions (a), (b) of $\S 4$. In fact, we see that, in (b), the points of $U_{v}$ may be separated by suitable ratios of Poincaré-Eisenstein series. From this follows, as in [2], [3], [16],

THEOREM 3. The compactification $V^{*}$ of $V=X / \Gamma$ carries a natural analytic structure, compatible with the analytic structure on any rational 
boundary component (including $X$ ), with which it becomes a normal analytic space. The normal analytic space $V^{*}$ can be projectively embedded as a projectively normal algebraic variety by means of a set of automorphic forms for $\Gamma$, of some suitable high weight.

It is easily seen that if $\operatorname{dim} G>3$, then $V^{*}-V$ has complex codimension $\geqq 2$. Making use of Levi's and H. Kneser's theorems on removable singularities of holomorphic or meromorphic functions, we derive from Theorem 3 the following corollaries:

Corollary 1. Let $\operatorname{dim} G>3$. Then the field of meromorphic functions on $V=X / \Gamma$ is an algebraic function field of transcendence degree equal to $\operatorname{dim}_{C} X$. Any such function is the quotient of automorphic forms of the same weight.

We recall that we have assumed $G$ to be simple over $Q$. However, Theorem 3 is valid without any such restriction, and Corollaries 1 and 2 are valid if $G$ has no invariant subgroup defined over $\boldsymbol{Q}$ of dimension three.

Corollary 2. Let $\operatorname{dim} G>3$. If $f$ is an automorphic form of even weight, its limit (in the sense mentioned above for Poincaré-Eisenstein series) on any rational boundary component exists. The space of automorphic forms of a given weight $m>0$ is finite-dimensional.

Corollary 1 and the finite-dimensionality of the space of automorphic forms may also be proved by showing that $\Gamma$ is pseudo-concave in the sense of Andreotti-Grauert [1]; this approach will be discussed elsewhere by one of the authors of the present note. The first part of Corollary 2, which is an extension of Koecher's theorem, can also be proved, independently of Theorem 3, by the methods of [11, pp. 134143], together with some of the results of [5] giving conditions for the finiteness of the volume of $H_{R} / H_{Z}$ for an algebraic group $H$ over Q. Moreover, we note that Corollary 2 extends to vector-valued automorphic forms, with a suitable automorphy factor of the type considered in [16]. This follows from Theorem 3, and from an unpublished result of J.-P. Serre which, together with [6], implies that if $A$ is the sheaf of germs of such automorphic forms, then the direct image $i_{*} A$ of $A$, with respective to the inclusion $i: V \rightarrow V^{*}$, is an algebraic coherent sheaf on $V^{*}$. The finite-dimensionality is also of course a consequence of pseudo-concavity.

\section{REFERENCES}

1. A. Andreotti and H. Grauert, Algebraische Körper von automorphen Funktionen, Nachr. Akad. Wiss. Göttingen Math.-Phys. K1. II (1961), 39-48. 
2. W. L. Baily, Jr., Satake's compactification of $V_{n}$, Amer. J. Math. 80 (1958), 348-364.

3. - On the Hilbert-Siegel modular space, Amer. J. Math. 81 (1959), 846-874.

4. A. Borel, Ensembles fondamentaux pour les groupes arithmétiques, Colloque sur la théorie des groupes algébriques, CBRM, Brussels (1962), 23-40.

5. A. Borel and Harish-Chandra, Arithmetic subgroups of algebraic groups, Ann. of Math. (2) 75 (1962), 485-535.

6. H. Cartan, Fonctions automorphes et séries de Poincaré, J. Analyse Math. 6 (1958), 169-175.

7. Harish-Chandra, Representations of semi-simple Lie groups. VI, Amer. J. Math. 78 (1956), 564-628

8. S. Helgason, Differential geometry and symmetric spaces, Academic Press, New York, 1962.

9. A. Korányi and J. Wolf, Generalized Cayley transforms of bounded symmetric domains, Ann. of Math. (to appear).

10. C. C. Moore, Compactifications of symmetric spaces. II. The Cartan domains, Amer. J. Math. (to appear).

11. I. I. Pyateckir-Shapiro, The geometry of the classical domains and the theory of automorphic functions, Gosizdat, Moscow, 1961. (Russian)

12. R. Remmert and K. Stein, Ueber die wesentlichen Singularitäten analytischer Mengen, Math. Ann. 126 (1953), 263-306.

13. I. Satake, On the compactification of the Siegel space, J. Indian Math. Soc. 20 (1956), 259-281.

14. - On representations and compactifications of symmetric spaces, Ann. of Math. (2) 71 (1960), 77-110.

15. - On compactifications of the quotient spaces for arithmetically defined discontinuous groups, Ann. of Math. (2) 72 (1960), 555-580.

16. Seminaire Henri Cartan, 10ème année, Fonctions Automorphes (multigraphé), Paris, 1958, 2 volumes.

University of Chicago,

The Institute for Advanced Study and

UnIversity of Paris 